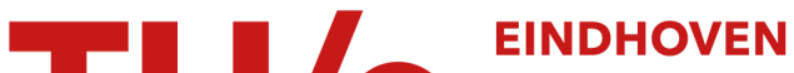 UNIVERSITY OF TECHNOLOGY
}

\section{Existence of weak solutions for the Mullins-Sekerka flow}

Citation for published version (APA):

Röger, M. (2005). Existence of weak solutions for the Mullins-Sekerka flow. SIAM Journal on Mathematical Analysis, 37(1), 291-301. https://doi.org/10.1137/S0036141004439647

DOI:

$10.1137 / \mathrm{S} 0036141004439647$

Document status and date:

Published: 01/01/2005

\section{Document Version:}

Publisher's PDF, also known as Version of Record (includes final page, issue and volume numbers)

\section{Please check the document version of this publication:}

- A submitted manuscript is the version of the article upon submission and before peer-review. There can be important differences between the submitted version and the official published version of record. People interested in the research are advised to contact the author for the final version of the publication, or visit the $\mathrm{DOI}$ to the publisher's website.

- The final author version and the galley proof are versions of the publication after peer review.

- The final published version features the final layout of the paper including the volume, issue and page numbers.

Link to publication

\section{General rights}

Copyright and moral rights for the publications made accessible in the public portal are retained by the authors and/or other copyright owners and it is a condition of accessing publications that users recognise and abide by the legal requirements associated with these rights.

- Users may download and print one copy of any publication from the public portal for the purpose of private study or research.

- You may not further distribute the material or use it for any profit-making activity or commercial gain

- You may freely distribute the URL identifying the publication in the public portal.

If the publication is distributed under the terms of Article 25fa of the Dutch Copyright Act, indicated by the "Taverne" license above, please follow below link for the End User Agreement:

www.tue.nl/taverne

Take down policy

If you believe that this document breaches copyright please contact us at:

openaccess@tue.nl

providing details and we will investigate your claim. 


\title{
EXISTENCE OF WEAK SOLUTIONS FOR THE MULLINS-SEKERKA FLOW*
}

\author{
MATTHIAS RÖGER ${ }^{\dagger}$
}

\begin{abstract}
The long-time existence of solutions for the Mullins-Sekerka problem in a new weak formulation is proved. Using a variational approach introduced by Luckhaus and Sturzenhecker [Calc. Var. Partial Differential Equations, 3 (1995), pp. 253-271], time-discrete solutions are constructed, satisfying approximate Gibbs-Thomson laws in a BV-formulation. But since the passage to a limit allows a loss of surface area for the phase interfaces, convergence in this setting is in general not true. We consider the surface measure of the phase interfaces and use the theory of varifolds to obtain a rigorous passage to a limit in a suitable weak formulation of the Gibbs-Thomson law.
\end{abstract}

Key words. free boundaries, Mullins-Sekerka flow, varifolds

AMS subject classifications. 49Q20, 80A22

DOI. $10.1137 /$ S0036141004439647

1. Introduction. The Mullins-Sekerka flow is a variant of the classical Stefan problem and describes phase transitions, such as melting or solidification processes, where a negligible specific heat of the material under consideration can be assumed. In this situation the energy balance is expressed by a quasi-stationary parabolic equation. A geometric condition on the phase interface, known as Gibbs-Thomson law, accounts for surface tension effects. In contrast to the classical Stefan problem, the MullinsSekerka flow allows for superheating and undercooling, i.e., temperatures above the melting point in the solid phase or temperatures below the melting point in the liquid phase.

To state the problem we consider a given time interval $(0, T)$ and an open bounded region $\Omega \subset \mathbb{R}^{3}$ representing the body of the material. Denote $\Omega_{T}:=(0, T) \times \Omega$. The state variables are the relative temperature

$$
u: \Omega_{T} \rightarrow \mathbb{R}
$$

( $u=0$ denoting the melting point) and a phase function

$$
\mathcal{X}: \Omega_{T} \rightarrow\{0,1\},
$$

which partitions $\Omega$ at a time $t \in(0, T)$ into a liquid phase $\{\mathcal{X}(t,)=1$.$\} and a solid$ phase $\{\mathcal{X}(t,)=0$.$\} separated by the phase interface, that is, their common boundary$ in $\Omega$. The energy balance for the classical Stefan problem reads

$$
\partial_{t}(c u+L \mathcal{X})-k \Delta u=f
$$

in the sense of distributions, with a given heat source $f: \Omega \rightarrow \mathbb{R}$ and phase independent constants $c, L$, and $k$ describing the specific heat, latent heat, and heat capacity,

* Received by the editors January 9, 2004; accepted for publication (in revised form) December 28, 2004; published electronically October 7, 2005. This work was supported by DFG Sonderforschungsbereich 611 and by the European Community's Human Potential Programme under contract HPRNCT-2002-00274, FRONTS-SINGULARITIES.

http://www.siam.org/journals/sima/37-1/43964.html

${ }^{\dagger}$ Department of Mathematics and Computing Science, Eindhoven University of Technology, Den Dolech 2, 5600 MB Eindhoven, The Netherlands (mroeger@win.tue.nl). 
respectively. Assuming that $c$ is negligibly small and setting for simplicity $L=k=1$, we get the quasi-stationary energy balance

$$
\partial_{t} \mathcal{X}-\Delta u=f
$$

In the classical Stefan problem the temperature at the phase interface equals the melting temperature, that is,

$$
u(t, .)=0 \quad \text { on the phase interface. }
$$

Surface tension effects are taken into consideration by the Gibbs-Thomson law

$$
H(t, .)=u(t, .) \quad \text { on the phase interface, }
$$

where $H(t,$.$) denotes the scalar mean curvature of the phase interface, which we take$ as positive for convex liquid phases.

The Mullins-Sekerka problem is given by (1.2) and (1.4). An initial condition for $\mathcal{X}$ and a boundary condition for $u$ on $\partial \Omega$ are prescribed. This model can be seen as a quasi-stationary variant of the Stefan problem with Gibbs-Thomson law given by (1.1) and (1.4). Existence of classical solutions for the Mullins-Sekerka problem locally in time was proved by Chen, Hong, and Li [CHY96] and by Escher and Simonett [ES97]. In general, classical solutions can develop singularities. To derive long-time existence results one has to turn to weak formulations. Chen [Che96] obtains solutions globally in time studying the limit of a certain Cahn-Hilliard model. Here the Gibbs-Thomson law is satisfied in a rather weak and complex formulation. In particular, the measures giving the energy density are not necessarily rectifiable.

Luckhaus and Sturzenhecker give in [LS95] another weak formulation of the Mullins-Sekerka problem. Phase and temperature function

$$
\mathcal{X} \in \mathrm{L}^{\infty}(0, T ; \mathrm{BV}(\Omega ;\{0,1\})), \quad u \in \mathrm{L}^{2}\left(0, T ; \mathrm{H}^{1,2}(\Omega)\right)
$$

satisfy (1.2) in the sense of distributions and (1.4) in the BV-formulation of the GibbsThomson law, that is,

$$
\int_{0}^{T} \int_{\Omega}\left(\nabla \cdot \xi-\frac{\nabla \mathcal{X}}{|\nabla \mathcal{X}|} \cdot D \xi \frac{\nabla \mathcal{X}}{|\nabla \mathcal{X}|}\right)(t, .)|\nabla \mathcal{X}|(t, .) d t=\int_{\Omega_{T}} \nabla \cdot(u \xi) \mathcal{X}
$$

for all $\xi \in C_{c}^{\infty}\left(\Omega_{T} ; \mathbb{R}^{3}\right)$.

Whereas this weak formulation is comparatively simple, the convergence of timediscrete approximations $\mathcal{X}^{h}$ and $u^{h}$ to correct weak solutions of (1.2) and (1.4) is only shown under an additional condition on the approximations, which reads

$$
\lim _{h \rightarrow 0} \int_{\Omega_{T}}\left|\nabla \mathcal{X}^{h}\right| \rightarrow \int_{\Omega_{T}}|\nabla \mathcal{X}| .
$$

This excludes a loss of surface area for the phase interfaces and allows one to prove the convergence of approximate Gibbs-Thomson laws within the BV-formulation.

In the present paper we use the time-discrete approximation scheme of [LS95] but drop condition (1.5). Difficulties which arise are captured in the following timeindependent example. Assume two solid parts of approximations $\mathcal{X}^{h}$ which merge to one when letting $h \rightarrow 0$. 

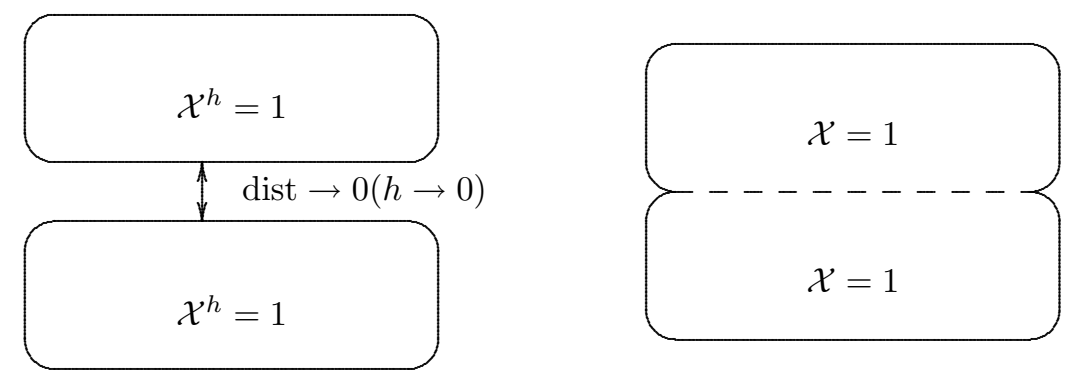

A part of the boundary, indicated by the dashed line, has ceased to separate two different phases. We call this part the hidden boundary, whereas the phase interface represents the physically relevant part of the boundary. Cusp singularities occur due to the cancellation of phase interfaces. As shown in [Sch97], the BV-formulation of the Gibbs-Thomson law breaks down.

These difficulties are tackled in [Rög03] and [Rög04], where the Stefan problem with Gibbs-Thomson law is treated. Following an idea of Schätzle [Sch01] we consider the surface measure of the phase interfaces to master possible cancellations. In the above example the surface measures $\left|\nabla \mathcal{X}^{h}\right|$ converge with $h \rightarrow 0$ to a Radon measure that has double multiplicity on the hidden boundary. This suggests the use of the concept of integral varifolds introduced by Almgren [Alm65]. Geometric measure theory provides a notion of mean curvature for integral varifolds; Schätzle [Sch01] investigates the convergence of approximate Gibbs-Thomson equations in this context. However, the control about the hidden boundaries is quite weak and we have to focus on the physically relevant part of the boundary. For this purpose the following Proposition, which we have proved in an earlier work, will be crucial (for the notations, consult section 2).

Proposition 1.1 (see [Rög04, Proposition 3.1]). Let $\Omega \subset \mathbb{R}^{n}$ be open, $E \subset \Omega$, and $\mathcal{X}_{E} \in \mathrm{BV}(\Omega)$. Assume that there are two integral $(n-1)$-varifolds $\mu_{1}, \mu_{2}$ on $\Omega$ such that for $i=1,2$ the following hold:

$$
\begin{gathered}
\partial^{*} E \subset \operatorname{spt}\left(\mu_{i}\right), \\
\mu_{i} \text { has locally bounded first variation with mean curvature vector } \vec{H}_{\mu_{i}}, \\
\vec{H}_{\mu_{i}} \in \mathrm{L}_{\mathrm{loc}}^{s}\left(\mu_{i}\right), s>n-1, s \geq 2 .
\end{gathered}
$$

Then

$$
\left.\vec{H}_{\mu_{1}}\right|_{\partial^{*} E}=\left.\vec{H}_{\mu_{2}}\right|_{\partial^{*} E}
$$

is satisfied $\mathcal{H}^{n-1}$-almost everywhere on $\partial^{*} E$.

This proposition justifies the following definition.

Definition 1.2. Let $E \subset \Omega$ and $\mathcal{X}_{E} \in \mathrm{BV}(\Omega)$, and assume that there exists an integral $(n-1)$-varifold $\mu$ on $\Omega$ satisfying $(1.6)-(1.8)$. Then we call

$$
\vec{H}:=\left.\vec{H}_{\mu}\right|_{\partial^{*} E}
$$

the generalized mean curvature vector of $\partial^{*} E$ and define a scalar mean curvature by

$$
H:=\vec{H} \cdot \frac{\nabla \mathcal{X}}{|\nabla \mathcal{X}|} \text { on } \partial^{*} E \text {. }
$$


The essential boundary $\partial^{*} E$ represents the phase interface, whereas $\operatorname{spt}(\mu) \backslash \partial^{*} E$ can be seen as a hidden boundary. Proposition 1.1 shows that the varifold's mean curvature restricted to the phase interface is a property of the phase interface itself and independent of the location of hidden boundaries.

Our solutions of the Mullins-Sekerka problem satisfy the Gibbs-Thomson in the sense that for almost all times a generalized mean curvature for the phase interface exists and is given pointwise almost everywhere by (1.4).

THEOREM 1.3. Let $\Omega \subset \mathbb{R}^{3}$ be an open bounded set with Lipschitz boundary, $\Gamma_{D}$ a $\mathcal{H}^{2}$-measurable subset of $\partial \Omega$ with $\mathcal{H}^{2}\left(\Gamma_{D}\right)>0$, and define $M_{0}:=\left\{v \in \mathrm{H}^{1,2}(\Omega): v=0\right.$ on $\left.\Gamma_{D}\right\}$. For given data

$$
\begin{aligned}
\mathcal{X}_{0} & \in \mathrm{BV}(\Omega ;\{0,1\}), \\
u_{D} & \in \mathrm{H}^{1,2}(\Omega), \\
f & \in \mathrm{L}^{2}(\Omega),
\end{aligned}
$$

there exists functions

$$
\begin{gathered}
\mathcal{X} \in \mathrm{L}^{\infty}(0, T ; \mathrm{BV}(\Omega ;\{0,1\})), \\
u \in \mathrm{L}^{2}\left(0, T ; u_{D}+M_{0}\right),
\end{gathered}
$$

such that

$$
\int_{\Omega_{T}} \mathcal{X} \partial_{t} \varphi+\int_{\Omega} \mathcal{X}_{0} \varphi(0, .)-\int_{\Omega_{T}} \nabla u \cdot \nabla \varphi=-\int_{\Omega_{T}} f \varphi
$$

for all $\varphi \in C_{c}^{\infty}([0, T) \times \bar{\Omega}), \varphi=0$ on $\Gamma_{D}$. For almost all $t \in(0, T)$ a generalized mean curvature $H(t)$ of $\partial^{*}\{\mathcal{X}(t,)=1$.$\} exists and satisfies \mathcal{H}^{2}$-almost everywhere on $\partial^{*}\{\mathcal{X}(t,)=1\}:$.

$$
H(t, .)=u(t, .)
$$

As shown in [Rög04], (1.10) is well defined since for $u \in \mathrm{L}^{2}\left(0, T ; \mathrm{H}^{1,2}(\Omega)\right)$ trace values of $u(t,$.$) exist \mathcal{H}^{2}$-almost everywhere for almost all $t \in(0, T)$. Furthermore, our formulation of the Gibbs-Thomson law generalizes the BV-formulation. Nevertheless we remark that this solution concept does not include a weak formulation of a boundary condition on the angle between the phase interface and the fixed boundary $\partial \Omega$ (see also the comments in [Rög03]). Moreover, since our focus is on the validation of the Gibbs-Thomson law, we have restricted our investigation to the case of a nonvanishing Dirichlet boundary. In the case of a pure Neumann boundary condition one has to take care of Lagrange multipliers occurring in the approximate GibbsThomson laws. For a discussion of this topic we refer to [BGS98], where a multiphase Mullins-Sekerka system under a pure Neumann boundary condition is investigated.

The proof of Theorem 1.3 is given in sections 3 and 4. Compared to the Stefan problem with Gibbs-Thomson law, the technical difficulty is due to the degeneracy of the energy balance equation and the lack of $\mathrm{L}^{1}\left(\Omega_{T}\right)$-compactness for the approximate temperature functions.

2. General definitions and notations. We fix some notations and basic definitions. As a general reference for geometric measure theory, see the book by Simon [Sim83]. 
For functions depending on time and space variables we denote " $\nabla$ " as the spatial gradient and " $\nabla \cdot$ " as the spatial divergence. For a differentiable function $f: \mathbb{R}^{n} \rightarrow \mathbb{R}^{n}$ and a $k$-dimensional subspace $T$ of $\mathbb{R}^{n}$ we define the divergence restricted to $T$ as

$$
\operatorname{div}_{T} f(x):=\sum_{i=1}^{k} t_{i} \cdot D f(x) t_{i}
$$

where $\left\{t_{i}\right\}_{i=1, \ldots, k}$ is any orthonormal basis of $T$.

For $\Omega \subset \mathbb{R}^{n}$ open, $\mu$ a Radon measure on $\Omega, x \in \Omega$, and $\varrho>0$, define the scaled measures

$$
\mu_{x, \varrho}(A):=\varrho^{-n+1} \mu(x+\varrho A) .
$$

A $k$-dimensional subspace $P \subset \mathbb{R}^{n}$ is called the $k$-dimensional tangential plane of $\mu$ in $x$, denoted by $T_{x} \mu$, if there is $\theta>0$ such that

$$
\mu_{x, \varrho} \rightarrow \theta \mathcal{H}^{k}\lfloor P \text { as Radon measures }
$$

as $\varrho$ tends to zero. In this case $\theta$ is the multiplicity of $\mu$ in $x$.

We call $\mu$ a rectifiable $(n-1)$-varifold if for $\mu$-almost all $x \in \Omega$ the $(n-1)$ dimensional tangential plane $T_{x} \mu$ exists, and an integral $(n-1)$-varifold if in addition the multiplicity of $\mu$ is $\mu$-almost everywhere integer-valued. A general $(n-1)$-varifold is a Radon measure on the Grassmannian $G^{n-1} \Omega$, which denotes the product of $\Omega$ and the space of $(n-1)$-dimensional subspaces of $\mathbb{R}^{n}$. In the present paper we identify a rectifiable $(n-1)$-varifold $\mu$ and the related Radon measure $V_{\mu}$ on $G^{n-1} \Omega$, defined by

$$
V_{\mu}(\zeta):=\int_{\Omega} \zeta\left(x, T_{x} \mu\right) d \mu(x) \quad \text { for } \zeta \in C_{c}^{0}\left(G^{n-1} \Omega\right)
$$

The first variation of a rectifiable $(n-1)$-varifold $\mu$ is given by

$$
\delta \mu(\xi):=\int_{\Omega} \operatorname{div}_{T_{x} \mu} \xi(x) d \mu(x) \quad \text { for } \xi \in C_{c}^{1}\left(\Omega ; \mathbb{R}^{n}\right) .
$$

We say that $\mu$ is of locally bounded first variation with mean curvature vector $\vec{H}_{\mu}$ if $\vec{H}_{\mu} \in \mathrm{L}_{l o c}^{1}(\mu)$ and

$$
\delta \mu(\xi)=\int_{\Omega}-\vec{H}_{\mu} \cdot \xi d \mu \quad \text { for all } \xi \in C_{c}^{1}\left(\Omega ; \mathbb{R}^{n}\right) .
$$

For a $\mathcal{L}^{n}$-measurable set $E \subset \Omega$ of finite perimeter let $\partial^{*} E$ denote the reduced boundary of $E$ in $\Omega$, that is, the subset of $\partial E \cap \Omega$ where a generalized inner normal exists as Radon-Nikodým derivative $\nabla \mathcal{X}_{E} /\left|\nabla \mathcal{X}_{E}\right|$ with length one. Then $\left|\nabla \mathcal{X}_{E}\right|=\mathcal{H}^{n-1}\left\lfloor\partial^{*} E\right.$ is an integral (with density 1$)(n-1)$-varifold on $\Omega$ (see [AFP00, paragraph 3.5]).

3. Time discretization. We use the scheme introduced by Luckhaus and Sturzenhecker [LS95] to construct time-discrete approximations. Given a time step $h>0$, determine iteratively step functions in time

$$
u^{h}:(0, T) \rightarrow\left(u_{\mathrm{D}}+M_{0}\right), \quad \mathcal{X}^{h}:(0, T) \rightarrow \mathrm{BV}(\Omega ;\{0,1\})
$$


by the following procedure. We use the abbreviations $\mathcal{X}_{t}^{h}=\mathcal{X}^{h}(t,),. u_{t}^{h}=u^{h}(t,$. and set

$$
\mathcal{X}_{t}^{h}:=\mathcal{X}_{0} \quad \text { for } 0 \leq t<h .
$$

For known $u_{t-h}^{h}, \mathcal{X}_{t-h}^{h}$ we define functionals $F_{t}^{h}: \operatorname{BV}(\Omega ;\{0,1\}) \rightarrow \mathbb{R}$,

$$
F_{t}^{h}(\mathcal{X}):=\int_{\Omega}|\nabla \mathcal{X}|+\frac{1}{2} \int_{\Omega} B^{h}\left(\mathcal{X}-\mathcal{X}_{t-h}^{h}-h f\right)\left(\mathcal{X}-\mathcal{X}_{t-h}^{h}-h f\right),
$$

where for $v \in \mathrm{L}^{2}(\Omega)$ the function $B^{h}(v)$ denotes the solution of

$$
-h \Delta B^{h}(v)=v,\left.\quad B^{h}(v)\right|_{\Gamma_{D}}=u_{\mathrm{D}},\left.\quad \nabla B^{h}(v) \cdot \nu_{\Omega}\right|_{\partial \Omega \backslash \Gamma_{D}}=0 .
$$

Let $\mathcal{X}_{t}^{h}$ be a global minimizer of $F_{t}^{h}$ and define

$$
u_{t}^{h}:=-B^{h}\left(\mathcal{X}_{t}^{h}-\mathcal{X}_{t-h}^{h}-h f\right) .
$$

(3.3) yields the approximate energy-balance

$$
\partial_{t}^{-h} \mathcal{X}^{h}=\Delta u^{h}+f .
$$

Since the first variation of $F_{t}^{h}$ vanishes in $\mathcal{X}_{t}^{h}$, we get for all $\xi \in C_{c}^{1}\left(\Omega, \mathbb{R}^{3}\right)$

$$
\int_{\Omega}\left(\nabla \cdot \xi-\frac{\nabla \mathcal{X}_{t}^{h}}{\left|\nabla \mathcal{X}_{t}^{h}\right|} \cdot D \xi \frac{\nabla \mathcal{X}_{t}^{h}}{\left|\nabla \mathcal{X}_{t}^{h}\right|}\right)\left|\nabla \mathcal{X}_{t}^{h}\right|=\int_{\Omega} \mathcal{X}_{t}^{h} \nabla \cdot\left(u_{t}^{h} \xi\right)
$$

which is the BV-formulation of the approximate Gibbs-Thomson law

$$
H_{t}^{h}=u_{t}^{h}
$$

where $H_{t}^{h}$ denotes the mean curvature of $\partial^{*}\left\{\mathcal{X}_{t}^{h}=1\right\}$.

To derive compactness properties for the approximate solutions we first observe $B^{h}(h v)=B^{1}(v)$ and

$$
\begin{aligned}
\int_{\Omega} B^{h}(v) v & =h \int_{\Omega}\left|\nabla B^{h}(v)\right|^{2}-h \int_{\Omega} \nabla B^{h}(v) \cdot \nabla u_{\mathrm{D}}+\int_{\Omega} v u_{\mathrm{D}} \\
& \geq \frac{h}{2} \int_{\Omega}\left|\nabla B^{h}(v)\right|^{2}-\frac{h}{2} \int_{\Omega}\left|\nabla u_{\mathrm{D}}\right|^{2}+\int_{\Omega} v u_{\mathrm{D}} .
\end{aligned}
$$

With $F_{\tau}^{h}\left(\mathcal{X}_{\tau}^{h}\right) \leq F_{\tau}^{h}\left(\mathcal{X}_{\tau-h}^{h}\right)$, we calculate for all $0<\tau<T$

$$
\begin{aligned}
& \int_{\Omega}\left|\nabla \mathcal{X}_{\tau}^{h}\right|+\frac{h}{4} \int_{\Omega}\left|\nabla u_{\tau}^{h}\right|^{2}-\frac{h}{4} \int_{\Omega}\left|\nabla u_{\mathrm{D}}\right|^{2}+\frac{1}{2} \int_{\Omega}\left(\mathcal{X}_{\tau}^{h}-\mathcal{X}_{\tau-h}^{h}-h f\right) u_{\mathrm{D}} \\
\leq & \int_{\Omega}\left|\nabla \mathcal{X}_{\tau-h}^{h}\right|+\frac{h}{2} \int_{\Omega} B^{1}(f) f .
\end{aligned}
$$

Now, for any $t \in(0, T), t \in[M h,(M+1) h)$ with $M \in \mathbb{N}_{0}$, we take inequality (3.6) for $\tau_{i}=i h$ and sum over $i=1, \ldots, M$, which yields

$$
\begin{aligned}
& \int_{\Omega}\left|\nabla \mathcal{X}_{t}^{h}\right|+\frac{1}{4} \int_{0}^{t} \int_{\Omega}\left|\nabla u^{h}\right|^{2} \\
\leq & \int_{\Omega}\left|\nabla \mathcal{X}_{0}\right|+\frac{T}{4} \int_{\Omega}\left|\nabla u_{\mathrm{D}}\right|^{2}+\frac{1}{2} \int_{\Omega}\left|u_{\mathrm{D}}\right|(1+T|f|)+\frac{T}{2} \int_{\Omega} B^{1}(f) f .
\end{aligned}
$$


In particular we get

$$
\sup _{h>0}\left\|\mathcal{X}^{h}\right\|_{\mathrm{L}^{\infty}(0, T ; \operatorname{BV}(\Omega))}<\infty, \quad \sup _{h>0}\left\|u^{h}\right\|_{\mathrm{L}^{2}\left(0, T ; \mathrm{H}^{1,2}(\Omega)\right)}<\infty
$$

and Fatou's lemma ensures

$$
\liminf _{h \rightarrow 0}\left(t \mapsto\left\|u^{h}(t, .)\right\|_{\mathrm{H}^{1,2}(\Omega)}\right) \in \mathrm{L}^{2}(0, T) .
$$

In [LS95] an estimate for time differences of $\mathcal{X}^{h}$ is derived from (3.8); that is,

$$
\int_{\tau}^{T} \int_{\Omega}\left|\mathcal{X}_{t}^{h}-\mathcal{X}_{t-\tau}^{h}\right| d \mathcal{L}^{3} d t \leq C \tau^{\frac{1}{4}}
$$

holds for all $0<\tau<T$. The Fréchet-Kolmogorov-Riesz theorem (see, for example, [DS88, IV.8, Theorem 21]), the weak*-compactness of $\mathrm{L}^{2}\left(0, T ; \mathrm{H}^{1,2}(\Omega)\right)$, and (3.8), (3.9), and (3.10) yield the following proposition.

Proposition 3.1. There exists a subsequence $h \rightarrow 0$ and functions $\mathcal{X} \in$ $\mathrm{L}^{\infty}(0, T ; \mathrm{BV}(\Omega)), u \in \mathrm{L}^{2}\left(0, T ; u_{D}+M_{0}\right)$, such that

$$
\begin{gathered}
u^{h} \rightarrow u \text { weakly in } \mathrm{L}^{2}\left(0, T ; \mathrm{H}^{1,2}(\Omega)\right), \\
\mathcal{X}^{h} \rightarrow \mathcal{X} \quad \text { in } \mathrm{L}^{1}\left(\Omega_{T}\right),
\end{gathered}
$$

and for almost all $t \in(0, T)$ we have

$$
\begin{gathered}
\mathcal{X}^{h}(t) \rightarrow \mathcal{X}(t) \quad \text { in } \mathrm{L}^{1}(\Omega), \\
\sup _{h>0}\left\|\mathcal{X}^{h}(t, .)\right\|_{\mathrm{BV}(\Omega)}<\infty, \\
\liminf _{h \rightarrow 0}\left\|u^{h}(t, .)\right\|_{\mathrm{H}^{1,2}(\Omega)}<\infty .
\end{gathered}
$$

4. Convergence to solutions. Let $u, \mathcal{X}$ and a subsequence $h \rightarrow 0$ be given as in Proposition 3.1. To prove Theorem 1.3 we pass (3.4) and (3.5) to a limit and show $u, \mathcal{X}$ to be a correct weak solution of the Mullins-Sekerka problem. The energy balance is derived in a standard way. Use any $\varphi \in C_{c}^{\infty}([0, T) \times \bar{\Omega})$ with $\left.\varphi\right|_{\Gamma_{D}}=0$ as a test function in (3.4), perform a discrete partial integration, and use (3.11) and (3.12) to obtain (1.9).

The main effort is the passage to a limit in the approximate Gibbs-Thomson law (3.5). To use a convergence result of Schätzle [Sch01] we argue pointwise in time. Due to the lack of strong $\mathrm{L}^{1}\left(\Omega_{T}\right)$-compactness of the approximate temperatures, we have to consider any limit point of $\left(u^{h}(t, .)\right)_{h>0}$ in the weak- $\mathrm{H}^{1,2}(\Omega)$ topology and identify their traces on $\partial^{*}\{\mathcal{X}(t,)=1$.$\} with the trace of the weak limit u$ in (3.11).

Let us denote by $\mu_{t}^{h}$ the integral 2-varifolds with density one associated to the surface measure of the phase interfaces

$$
\mu_{t}^{h}(\eta):=\int_{\Omega} \eta\left|\nabla \mathcal{X}_{t}^{h}\right| \quad \text { for } \eta \in C_{c}^{0}(\Omega) .
$$

For the first variation of $\mu_{t}^{h}$ we obtain, recalling (3.5),

$$
\delta \mu_{t}^{h}(\xi)=\int_{\Omega}\left(\nabla \cdot \xi-\frac{\nabla \mathcal{X}_{t}^{h}}{\left|\nabla \mathcal{X}_{t}^{h}\right|} \cdot D \xi \frac{\nabla \mathcal{X}_{t}^{h}}{\left|\nabla \mathcal{X}_{t}^{h}\right|}\right)\left|\nabla \mathcal{X}_{t}^{h}\right|=\int_{\Omega} \mathcal{X}_{t}^{h} \nabla \cdot\left(u_{t}^{h} \xi\right)
$$


for all $\xi \in C_{c}^{1}\left(\Omega, \mathbb{R}^{3}\right)$. Finally, we define $T^{h}: \mathrm{L}^{2}\left(0, T ; C_{c}^{1}\left(\Omega, \mathbb{R}^{3}\right)\right)^{*} \rightarrow \mathbb{R}$ by

$$
T^{h}(\xi)=\int_{0}^{T} \delta \mu_{t}^{h}(\xi(t, .)) d t
$$

and observe from (3.5) and (3.8) that

$$
\begin{aligned}
\left\|\delta \mu_{t}^{h}\right\|_{C_{c}^{1}\left(\Omega, \mathbb{R}^{3}\right)^{*}} & \leq C(\Omega)\left\|u_{t}^{h}\right\|_{H^{1,2}(\Omega)}, \\
\left\|T^{h}\right\|_{L^{2}\left(0, T ; C_{c}^{1}\left(\Omega, \mathbb{R}^{3}\right)\right)^{*}} & \leq C^{\prime}(\Omega),
\end{aligned}
$$

where $C(\Omega), C^{\prime}(\Omega)$ are independent of $h>0$.

In a first step we will prove that the phase interfaces $\partial^{*}\{\mathcal{X}(t,)=1$.$\} have a$ generalized mean curvature. Due to Proposition 1.1 this mean curvature is determined by the phase interface itself and thus given by the strong convergence in (3.12) and (3.13). Even more, any limit of the first variations $\left(\delta \mu_{t}^{h}\right)_{h>0}$ is determined by (3.12).

Lemma 4.1. For almost all $t \in(0, T)$ the phase interface $\partial^{*}\{\mathcal{X}(t,)=1$.$\} has a$ generalized mean curvature $\vec{H}(t)$ in the sense of Definition 1.2 with

$$
\vec{H}(t) \in \mathrm{L}_{\text {loc }}^{4}(|\nabla \mathcal{X}(t, .)|) .
$$

For any subsequence $\left(h_{i}\right)_{i \in \mathbb{N}}, h_{i} \rightarrow 0(i \rightarrow \infty)$, with $\sup _{i \in \mathbb{N}}\left\|u^{h_{i}}(t, .)\right\|_{\mathrm{H}^{1,2}(\Omega)}<\infty$ and for all $\xi \in C_{c}^{1}\left(\Omega, \mathbb{R}^{3}\right)$ we obtain

$$
\delta \mu_{t}^{h_{i}}(\xi) \rightarrow \int_{\Omega}-\vec{H}(t) \cdot \xi|\nabla \mathcal{X}(t, .)| .
$$

Defining the functionals $T(t) \in C_{c}^{1}\left(\Omega, \mathbb{R}^{3}\right)^{*}$ by

$$
\langle\xi, T(t)\rangle:=\int_{\Omega}-\vec{H}(t) \cdot \xi|\nabla \mathcal{X}(t, .)|,
$$

we finally observe that

$$
T \in \mathrm{L}^{2}\left(0, T ; C_{c}^{1}\left(\Omega, \mathbb{R}^{3}\right)\right)^{*}
$$

holds.

Proof. Fix any $t \in(0, T)$ such that $(3.13)-(3.15)$ hold and let $\left(h_{i}\right)_{i \in \mathbb{N}}$ be an arbitrary subsequence with

$$
\sup _{i \in \mathbb{N}}\left\|u^{h_{i}}(t, .)\right\|_{\mathrm{H}^{1,2}(\Omega)}<\infty .
$$

Define $V^{h} \in C_{c}^{0}\left(G^{2} \Omega\right)^{*}$ as the general varifold associated to the density-one integer varifold $\left|\nabla \mathcal{X}^{h}(t,).\right|$. Recalling (3.14) and (4.8), there is a subsequence $\left(\tilde{h}_{i}\right)_{i \in \mathbb{N}}$, a function $v \in \mathrm{H}^{1,2}(\Omega)$, and a Radon measure $V \in C_{c}^{0}\left(G^{2} \Omega\right)^{*}$ such that

$$
\begin{array}{rlr}
u^{\tilde{h}_{i}}(t, .) & \rightarrow v & \text { weakly in } \mathrm{H}^{1,2}(\Omega), \\
V^{\tilde{h}_{i}} & \stackrel{*}{\rightarrow} V & \text { weakly* in } C_{c}^{0}\left(G^{2} \Omega\right)^{*} .
\end{array}
$$

With (3.5), (3.12), and (4.9), (4.10) all assumptions of Theorem 1.1 in [Sch01] are fulfilled and we obtain that

$$
V=V_{\mu} \quad \text { for an integral } 2 \text {-varifold } \mu,
$$

$\mu$ has locally bounded first variation with mean curvature vector $\vec{H}_{\mu}$,

$$
\vec{H}_{\mu} \in \mathrm{L}_{\mathrm{loc}}^{4}(\mu),
$$


and that

$$
\vec{H}_{\mu}=v \nu(t, .)
$$

holds $\mu$-almost everywhere, with

$$
\nu(t, .)= \begin{cases}\frac{\nabla \mathcal{X}(t, .)}{|\nabla \mathcal{X}(t, .)|} & \text { on } \partial^{*}\{\mathcal{X}(t, .)=1\}, \\ 0 & \text { elsewhere. }\end{cases}
$$

According to Definition 1.2 the phase interface $\partial^{*}\{\mathcal{X}(t,)=1$.$\} has the generalized$ mean curvature vector $\vec{H}(t,)=.\left.\vec{H}_{\mu}\right|_{\partial^{*}\{\mathcal{X}(t, .)=1\}}$ and (4.13) yields (4.4). Moreover, due to [Sch01, Theorem 1.2], we have

$$
\vec{H}(t, .)=0 \quad \mu \text {-almost everywhere in }\left\{\theta^{2}(\mu, .) \neq 1\right\} .
$$

From (4.10) and (4.11) we obtain the convergence of $\mu_{t}^{\tilde{h}_{i}}$ to $\mu$ and therefore also the convergence of their first variations. Using (4.12), (4.14), and (4.15) we calculate

$$
\begin{aligned}
\lim _{i \rightarrow \infty} \delta \mu_{t}^{\tilde{h}_{i}}(\xi) & =\delta \mu(\xi) \\
& =\int_{\Omega}-\vec{H}_{\mu} \cdot \xi d \mu=\int_{\Omega}-\vec{H}(t, .) \cdot \xi|\nabla \mathcal{X}(t, .)|=\langle\xi, T(t, .)\rangle .
\end{aligned}
$$

Since $\vec{H}(t)$ is determined by $\mathcal{X} \in \mathrm{BV}(\Omega ;\{0 ; 1\})$ the functional $T(t)$ does not depend on the choices of $v, V$, and $\left(\tilde{h}_{i}\right)$. Thus we deduce

$$
\delta \mu_{t}^{h_{i}} \stackrel{*}{*} T(t)
$$

for the whole sequence $\left(h_{i}\right)_{i \in \mathbb{N}}$ and for all subsequences of $h \rightarrow 0$ for which (4.8) holds. We choose $h_{i} \rightarrow 0$ such that

$$
\lim _{i \rightarrow \infty}\left\|u^{h_{i}}(t, .)\right\|_{\mathrm{H}^{1,2}(\Omega)}=\liminf _{h \rightarrow 0}\left\|u^{h}(t, .)\right\|_{\mathrm{H}^{1,2}(\Omega)}<\infty
$$

and the lower semicontinuity of the norm with respect to weak*-convergence, and thus (4.2) yields

$$
\begin{aligned}
\|T(t, .)\|_{C_{c}^{1}\left(\Omega ; \mathbb{R}^{3}\right)^{*}} & \leq \liminf _{i \rightarrow \infty}\left\|\delta \mu_{t}^{h_{i}}\right\|_{C_{c}^{1}\left(\Omega ; \mathbb{R}^{3}\right)^{*}} \\
& \leq \liminf _{i \rightarrow \infty} C(\Omega)\left\|u^{h_{i}}(t, .)\right\|_{\mathrm{H}^{1,2}(\Omega)} \\
& =C(\Omega) \liminf _{h \rightarrow 0}\left\|u^{h}(t, .)\right\|_{\mathrm{H}^{1,2}(\Omega)} .
\end{aligned}
$$

Recalling (3.9) we get $T \in \mathrm{L}^{2}\left(0, T ; C_{c}^{1}\left(\Omega ; \mathbb{R}^{3}\right)\right)^{*}$.

To prove that the mean curvature of the phase interface is given as trace of the weak limit $u$ in (3.11), the weak*-convergence of $T^{h}$ in $L^{2}\left(0, T ; C_{c}^{1}(\Omega)\right)^{*}$ will be crucial. The difficulty is that the pointwise convergence (4.5) holds only for time-dependent subsequences. We use an argument similar to a refined dominated convergence theorem in [PS93].

LEMma 4.2. There is a subsequence $h \rightarrow 0$ such that

$$
T^{h} \stackrel{*}{\rightarrow} T \quad \text { in } \mathrm{L}^{2}\left(0, T ; C_{c}^{1}\left(\Omega ; \mathbb{R}^{3}\right)\right)^{*} .
$$


Proof. Fix an arbitrary $\xi \in \mathrm{L}^{2}\left(0, T ; C_{c}^{1}\left(\Omega ; \mathbb{R}^{3}\right)\right)$. For $\alpha>0$ we define functions $T_{\alpha}^{h}:(0, T) \rightarrow C_{c}^{1}\left(\Omega ; \mathbb{R}^{3}\right)^{*}:$

$$
T_{\alpha}^{h}(t):=\left\{\begin{array}{lll}
\delta \mu_{t}^{h} & \text { if } & \left\|u^{h}(t, .)\right\|_{\mathrm{H}^{1,2}(\Omega)} \leq \alpha, \\
T(t) & \text { if } & \left\|u^{h}(t, .)\right\|_{\mathrm{H}^{1,2}(\Omega)}>\alpha .
\end{array}\right.
$$

For all $t \in(0, T)$, for which the assertions of Lemma 4.1 hold, the definition of $T_{\alpha}^{h}$ and (4.5) yield

$$
\left\langle\xi(t, .), T_{\alpha}^{h}(t)\right\rangle \rightarrow\langle\xi(t, .), T(t)\rangle \quad(h \rightarrow 0) .
$$

The estimate

$$
\left|\left\langle\xi(t, .), T_{\alpha}^{h}(t)\right\rangle\right| \leq\|\xi(t, .)\|_{C_{c}^{1}\left(\Omega ; \mathbb{R}^{3}\right)}\left(C \alpha+\|T(t)\|_{C_{c}^{1}\left(\Omega ; \mathbb{R}^{3}\right)^{*}}\right)
$$

and (4.7) give a $\mathrm{L}^{1}(0, T)$-dominator. Thus the Lebesgue theorem ensures

$$
\int_{0}^{T}\left\langle\xi(t, .), T_{\alpha}^{h}(t)\right\rangle d t \rightarrow \int_{0}^{T}\langle\xi(t, .), T(t)\rangle d t \quad(h \rightarrow 0) .
$$

Next, consider the sets $A^{h}:=\left\{t \in(0, T):\left\|u^{h}(t, .)\right\|_{\mathrm{H}^{1,2}(\Omega)}>\alpha\right\}$ and observe

$$
\begin{aligned}
& \left|\int_{0}^{T}\left\langle\xi(t, .), \delta \mu_{t}^{h}-T_{\alpha}^{h}(t)\right\rangle d t\right| \\
\leq & \int_{A^{h}}\left|\left\langle\xi(t, .), \delta \mu_{t}^{h}-T(t)\right\rangle\right| d t \\
\leq & \left(\int_{A^{h}}\|\xi(t, .)\|_{C_{c}^{1}\left(\Omega ; \mathbb{R}^{3}\right)}^{2} d t\right)^{\frac{1}{2}}\left(\left\|T^{h}\right\|_{L^{2}\left(0, T ; C_{c}^{1}\left(\Omega ; \mathbb{R}^{3}\right)^{*}\right)}+\|T\|_{L^{2}\left(0, T ; C_{c}^{1}\left(\Omega ; \mathbb{R}^{3}\right)^{*}\right)}\right) .
\end{aligned}
$$

By (4.3) and (4.7) the norms $\left\|T^{h}\right\|_{\mathrm{L}^{2}\left(0, T ; C_{c}^{1}\left(\Omega ; \mathbb{R}^{3}\right)^{*}\right)}$ and $\|T\|_{\mathrm{L}^{2}\left(0, T ; C_{c}^{1}\left(\Omega ; \mathbb{R}^{3}\right)^{*}\right)}$ are bounded uniformly in $h>0$. Estimating

$$
\left|A^{h}\right| \leq \frac{1}{\alpha^{2}}\left\|u^{h}\right\|_{\mathrm{L}^{2}\left(0, T ; \mathrm{H}^{1,2}(\Omega)\right)}^{2} \leq \frac{1}{\alpha^{2}} C
$$

by (3.8), we end up with

$$
\int_{0}^{T}\left\langle\xi(t, .), T^{h}-T_{\alpha}^{h}(t)\right\rangle d t \rightarrow 0 \quad(\alpha \rightarrow \infty)
$$

uniformly in $h>0$. Putting this together with (4.18) proves the lemma.

By establishing the Gibbs-Thomson law we finish the proof of Theorem 1.3.

Lemma 4.3. For all $\xi \in \mathrm{L}^{2}\left(0, T ; C_{c}^{1}\left(\Omega ; \mathbb{R}^{3}\right)\right)$ we obtain

$$
\int_{0}^{T}\langle\xi(t, .), T(t)\rangle d t=\int_{\Omega_{T}} \mathcal{X} \nabla \cdot(u \xi) .
$$

In particular, for almost all $t \in(0, T)$,

$$
H(t, .)=u(t, .)
$$

holds $\mathcal{H}^{2}$-almost everywhere on $\partial^{*}\{\mathcal{X}(t,)=1$.$\} .$ 
Proof. We get from (3.5), (3.11), (4.1) and Lemma 4.2

$$
\begin{aligned}
\int_{\Omega_{T}} \mathcal{X} \nabla \cdot(u \xi) & =\lim _{h \rightarrow 0} \int_{\Omega_{T}} \mathcal{X}^{h} \nabla \cdot\left(u^{h} \xi\right) \\
& =\lim _{h \rightarrow 0} \int_{0}^{T}\left\langle\xi(t, .), \delta \mu_{t}^{h}\right\rangle d t \\
& =\int_{0}^{T}\langle\xi(t, .), T(t)\rangle d t .
\end{aligned}
$$

Since no time derivative is involved we deduce that for almost all $t \in(0, T)$ and all $\xi \in C_{c}^{1}\left(\Omega ; \mathbb{R}^{3}\right)$,

$$
\int_{\Omega} \mathcal{X}(t, .) \nabla \cdot(u(t, .) \xi)=\langle\xi, T(t)\rangle
$$

holds. The Gauss-Green theorem [EG92, Theorem 5.8.1] and (4.6) yield

$$
\int_{\Omega}-u(t, .) \nu(t, .) \cdot \xi|\nabla \mathcal{X}(t, .)|=\int_{\Omega}-\vec{H}(t, .) \cdot \xi|\nabla \mathcal{X}(t, .)|
$$

with $\nu(t,)=.\nabla \mathcal{X}(t,) /.|\nabla \mathcal{X}(t,)$.$| on \partial^{*}\{\mathcal{X}(t,)=1$.$\} . This proves the Gibbs-Thomson$ law.

Acknowledgment. I thank R. Schätzle for many fruitful discussions and for referring me to geometric measure theory.

\section{REFERENCES}

[AFP00] L. Ambrosio, N. Fusco, and D. Pallara, Functions of Bounded Variation and Free Discontinuity Problems, Oxford Science Publications, Oxford, UK, 2000.

[Alm65] F. J. Almgren, The Theory of Varifolds, Princeton notes, Princeton, NJ, 1965.

[BGS98] L. Bronsard, H. Garcke, And B. Stoth, A multi-phase Mullins-Sekerka system: Matched asymptotic expansions and an implicit time discretisation for the geometric evolution problem, Proc. Roy. Soc. Edinburgh Sect. A, 128 (1998), pp. 481-506.

[Che96] X. Chen, Global asymptotic limit of solutions of the Cahn-Hilliard equation, J. Differential Geom., 44 (1996), pp. 262-311.

[CHY96] X. Chen, J. Hong, AND F. YI, Existence, uniqueness, and regularity of classical solutions of the Mullins-Sekerka problem, Comm. Partial Differential Equations, 21 (1996), pp. 1705-1727.

[DS88] N. Dunford And J. T. Schwartz, Linear Operators. Part I, John Wiley, New York, 1988.

[EG92] L. C. Evans And R. F. Gariepy, Measure Theory and Fine Properties of Functions, CRC Press, Boca Raton, FL, 1992.

[ES97] J. Escher And G. Simonett, Classical solutions for Hele-Shaw models with surface tension, Adv. Differential Equations, 2 (1997), pp. 619-642.

[LS95] S. LUCKHAUs AND T. STURZENHECKER, Implicit time discretization for the mean curvature flow equation, Calc. Var. Partial Differential Equations, 3 (1995), pp. 253-271.

[PS93] P. I. Plotnikov and V. N. Starovoitov, The Stefan problem with surface tension as a limit of a phase field model, Differential Equations, 29 (1993), pp. 395-404.

[Rög03] M. RöGER, Lösungen für das Stefan Problem mit Gibbs-Thomson Gesetz bei einer lokalen Minimierung, Ph.D. thesis, Universität Bonn, 2003.

[Rög04] M. RöGER, Solutions for the Stefan problem with Gibbs-Thomson law by a local minimisation, Interfaces Free Bound., 6 (2004), pp. 105-133.

[Sch97] R. Sснӓtzle, A counterexample for an approximation of the Gibbs-Thomson law, Adv. Math. Sci. Appl., 7 (1997), pp. 25-36.

[Sch01] R. SchätzLE, Hypersurfaces with mean curvature given by an ambient Sobolev function, J. Differential Geom., 58 (2001), pp. 371-420.

[Sim83] L. Simon, Lectures on Geometric Measure Theory, in Proceedings of the Centre for Mathematical Analysis, Vol. 3, Australian National University Canberra, 1983. 